\title{
DESEMPENHO DE HÍBRIDOS DE LINHAGENS PARCIALMENTE ENDOGÂMICAS DE MILHO EM REGIÕES DOS ESTADOS DE RORAIMA E MINAS GERAIS ${ }^{1}$
}

\author{
AGNALDO DONIZETE FERREIRA DE CARVALHO ${ }^{2}$ \\ JOÃO CÂNDIDO DE SOUZA ${ }^{3}$ \\ PEDRO HÉLIO ESTEVAM RIBEIRO ${ }^{4}$
}

\begin{abstract}
RESUMO - Entre as alternativas de tipos de híbridos de milho (Zea mays L.), os que envolvem linhagens parcialmente endogâmicas (LPE) $S_{2}$ possuem a vantagem de maior rapidez na obtenção, se comparados com linhagens de endogamia completa. Essas LPE $\mathrm{S}_{2}$ podem ser obtidas a partir de qualquer tipo de população, porém, uma boa opção seria aquelas derivadas de híbridos simples comerciais, que associam produtividade e grande proporção de locos segregantes. Com esse intuito, o presente trabalho foi conduzido visando à avaliação de híbridos de LPE $\mathrm{S}_{2}$ derivados de três híbridos simples comerciais. Para obtenção dos híbridos, foram selecionados $30 \mathrm{LPE}_{2}$ de três populações distintas das cultivares híbridas comerciais: AG-9012, C-333B e Z8392. Os cruzamentos foram realizados em esquema de dialelo circulante, em que cada LPE $S_{2}$ de uma população foi cruzada com outras três LPE $S_{2}$ de outra população. Para os cruzamentos, as LPE $\mathrm{S}_{2}$ foram semeadas em linha com dez metros cada uma. No aparecimento das primeiras espigas, as mesmas foram protegidas e, por ocasião da polinização, uma mistura de pólen de cada LPE $\mathrm{S}_{2}$ foi utilizada para polinizar pelo menos dez plantas de uma outra população LPE $\mathrm{S}_{2}$. Os híbridos obtidos foram avaliados em Boa Vista, Roraima e em Lavras, Minas Gerais, utilizando-se o delineamento lá-
\end{abstract}

tice simples $12 \times 12$, envolvendo os 135 híbridos LPE $\mathrm{S}_{2}$ obtidos e mais nove testemunhas, sendo elas as gerações $\mathrm{F}_{1}$ dos híbridos simples AG-9012, C-333B e Z8392, um híbrido duplo (C 435) e cinco outros híbridos intervarietais. A parcela experimental foi constituída por uma linha de três metros de comprimento, espaçada de $90 \mathrm{~cm}$ entre linhas e permanecendo um total de cinco plantas por metro linear após o desbaste. As seguintes variáveis foram avaliadas: peso de espiga despalhada (PESP), altura de planta (ALTP) e altura de espiga (ALTE). Para a PESP, $81 \%$ dos híbridos $\mathrm{S}_{2}$ foram superiores às médias das testemunhas. Observou-se que $17 \%$ dos híbridos de LPE $\mathrm{S}_{2}$ tiveram desempenho superior ao híbrido simples C-333B C (10,76 t/ha) e 46\% apresentaram desempenho superior à testemunha C-435 $(9,56$ t/ha), um híbrido duplo amplamente utilizado nos dois estados. Para ALTP e ALTE, os resultados foram semelhantes a PESP, salientado que o contraste $\mathrm{H}$ vs $\mathrm{T}$ foi não-significativo, inferindo-se que os híbridos $\mathrm{S}_{2} \mathrm{e}$ as testemunhas apresentaram, em média, as mesmas ALTP e ALTE. Com esse fato, evidencia-se que os híbridos LPE $S_{2}$ são semelhantes aos híbridos comerciais e mostra o potencial da utilização desse tipo de híbridos no Brasil, que pode substituir com vantagens alguns híbridos comerciais disponíveis no mercado.

TERMOS PARA INDEXAÇÃO: Milho híbrido, linhagem, dialelo, Zea mays.

\section{PERFORMANCE OF HYBRIDS OF PARTIALLY ENDOGAMIC LINES $S_{2}$ OF MAIZE IN REGIONS OF THE STATES OF RORAIMA AND MINAS GERAIS}

\footnotetext{
ABSTRACT - Among the types of maize hybrids those from $S_{2}$ families have the advantage of higher synthesis speed. $S_{2}$ families can be obtained from any

kind of population, but a good option are those derived from commercial single cross hybrids that associate productivity and many segregant loci. So, the objective
} 
of the research way to evaluate hybrids derived from $\mathrm{S}_{2}$ families descendant of three single cross hybrids. The selection of hybrids was atrained from $30 \mathrm{~S}_{2}$ families derived of three segregant populations descendent of the commercial hybrids AG- 9012, C-333B and Z8392. Three crossings were carried out using a circular partial diallel scheme, when each family from one population was crossed with three other families from other population. For the crossings the families had been sown, in line of $10 \mathrm{~m}$ long. When the first ear emerged, it was protected and during the pollination time a mixture of pollen of each family was used to pollinate at least ten plants of a family of an other population. The hybrids were evaluated in Boa Vista, Roraima state and Minas Gerais state, Brazil, using the simple lattice design $12 \times 12$, including 135 hybrids and nine checks. The checks were the F1 generation of the single cross hybrids AG 9012, C333 and Z 8392, a

INDEX TERMS: Hybrid maize, line, diallel, Zea mays.

\section{INTRODUÇÃO}

O maior sucesso no melhoramento da cultura do milho foi obtido por meio da obtenção de híbridos, que entraram na agricultura americana por volta de 1940 e, no Brasil, poucos anos depois. A partir de então, anualmente, são produzidas novas combinações híbridas, que possuem vantagens adicionais sobre as preexistentes e, em conseqüência, nesses quase sessenta anos ocorreu progresso genético expressivo na espécie (RUSSEL, 1991; DUVICK, 1994; ARAÚJO, 1995).

Em um programa de híbridos, a etapa mais onerosa e demorada é a obtenção e avaliação das linhagens (MIRANDA FILHO e VIÉGAS, 1987). Uma das alternativas para se acelerar o processo é a obtenção de híbridos a partir de linhagens parcialmente endogâmicas (LPE) $\mathrm{S}_{2}$. Nesse caso, grande parte dos locos já está em homozigose e gastam-se apenas duas gerações de autofecundação. Na literatura há relatos do emprego desse tipo de híbrido (SOUZA JÚNIOR, 1998); contudo, no Brasil, a sua utilização comercial não foi ainda relatada.

O emprego desse tipo de híbrido, além de acelerar o processo de obtenção, permitirá a redução no custo de produção de sementes híbridas com evidentes vantagens para os produtores. Com o aumento do número de linhagens, o número de combinações híbridas possíveis, dado pela fórmula $n(n-1) / 2$, torna-se inviável. Entretanto, existem novos métodos para identificação de combinações superiores (DANTAS, 1992). double cross hybrid (435) and five other intervariety hybrids. Each plot had one line of $3 \mathrm{~m}$ long, with five plants per linear meter, and spaced of $90 \mathrm{~cm}$. The fertilization and others cultural care were the usually employed in the maize culture. The evaluation was based on: weight of dehushed ear (WDE), plant height (PH) and ear height (EH). 81\% of the hybrids were superior to the cheeks based on WDE, $17 \%$ outyielded the single cross hybrid C333B (10.76 t/ha), and $46 \%$ were superior to C $435(9,56 \mathrm{t} / \mathrm{ha})$ which is a double cross hybrid widely used in both states. The results considering $\mathrm{PH}$ and $\mathrm{EH}$ were similar to WDE, however, the interaction hybrids $\mathrm{x}$ cheeks was not significant. Considering the results the $S_{2}$ hybrids were similar to the commercial hybrids point out the potential of those hybrids for using in Brazil for replacing with advantage same commercial hybrids.

\footnotetext{
Ciênc. agrotec., Lavras. V.27, n.5, p.985-990, set./out., 2003

A etapa que exige maior esforço é a de avaliação das combinações das linhagens $\mathrm{S}_{2}$, que necessariamente envolve a realização de cruzamentos biparentais. Inicialmente Davis (1927) e posteriormente Jenkins e Brunson (1932) sugeriram que as linhagens fossem avaliadas por "Topcrosses". No entanto, existe uma grande dificuldade experimental devido ao grande número de genótipos a serem avaliados. Uma alternativa seria a utilização do dialelo completo, porém, Dantas (1992) alerta para o grande número de combinações híbridas que terão que ser avaliadas. Com isso, o mesmo autor menciona a possibilidade do uso de dialelos parciais, em que apenas uma amostra aleatória de um total de cruzamentos é avaliada. Com esse método, pode-se avaliar o potencial de combinações de progênies provenientes de populações distintas, como: meios irmãos, irmãos germanos e linhagens parcialmente endogâmicas.

A produção de híbridos de milho a partir de linhagens $S_{2}$ há muito tempo já é vista como uma possibilidade. Mota (1984) avaliou 300 híbridos de progênies de meios irmãos das populações PiranãoVD2 e Piranão-VF1 e salientou a capacidade da utilização desses materiais, já que $87 \%$ dos híbridos apresentaram superioridade em relação à testemunha, Piranão-VD2. Diante desses resultados, o autor propôs que fossem produzidos esses tipos de híbrido em nível interpopulacional, ressalvando que híbridos de linhagens parcialmente endogâmicas devem apresentar as mesmas vantagens dos híbridos intervarie- 
tais, possuindo ainda a chance de serem mais produtivos e uniformes.

Davis (1927) observou que, depois de sucessivas avaliações endogâmicas, na geração $S_{2}$, já havia fixação de alguns caracteres, como produção de grãos em combinações híbridas. Além do mais, as linhagens parcialmente endogâmicas $S_{2}$, apresentaram produção de grãos $70 \%$ superior do que as suas derivadas $S_{5}$. Loeffel (1964) e Stangland e Russel (1981) demonstraram que cruzamentos $S_{2} \times S_{2}$ foram mais uniformes que híbridos duplos, além de apresentarem rendimento semelhante às suas derivadas completamente endogâmicas.

Carlone e Russel (1988) mencionaram que linhagens $\mathrm{S}_{2}$ foram mais produtivas, mais resistentes às variações ambientais, apresentaram menor interação com o ambiente e como desvantagem para a sua utilização, está a dificuldade de mantê-las.

Welhausen (1954) verificou que os primeiros híbridos duplos e triplos utilizados no México eram provenientes de linhagens parcialmente endogâmicas $S_{1}$. Medina (1990) conclui que a produção de híbridos a partir de linhagens $S_{2}$ foi uma alternativa barata e lucrativa para programas em desenvolvimento, após ter avaliado híbridos $S_{1} \times S_{1}$ a $S_{4} \times S_{4}$, e não ter encontrado diferenças significativas.

O presente trabalho foi conduzido em Boa Vista, Roraima e Lavras, Minas Gerais, visando à avaliação de híbridos de linhagens $S_{2}$ derivados de três híbridos simples comerciais.

\section{MATERIAL E MÉTODOS}

Para a obtenção dos híbridos, foram selecionadas 30 famílias $S_{2}$ de três populações derivadas de híbridos simples comerciais: AG 9012 (A), C-333B (B) e Z-8392 (C). Os cruzamentos foram realizados em esquema de dialelo circulante (CRUZ e REGAZZI, 1997), em que cada família de uma população foi cruzada com outras três famílias de outra população. Para os cruzamentos, as famílias foram semeadas em uma linha com dez metros de comprimento. No aparecimento das espigas, essas foram protegidas e, por ocasião da polinização, uma mistura de pólen de dez plantas (A) foi utilizada para polinizar pelo menos 10 plantas de uma família da outra população (B) ou (C). No mesmo campo, realizou-se o intercruzamento de 20 plantas de todas as famílias para sua manutenção.

Os híbridos obtidos foram avaliados no ano agrícola 2000/2001 em dois locais: Boa Vista, RR e Lavras, MG. Boa Vista está localizada a $02^{\circ} 54^{\prime} \mathrm{N}$ de latitude, $60^{\circ} 42^{\prime}$ W de longitude e a $92 \mathrm{~m}$ de altitude. Lavras está situada a $21^{\circ} 14^{\prime} \mathrm{S}$ de latitude, $40^{\circ} 17^{\prime} \mathrm{W}$ de longitude e
$914 \mathrm{~m}$ de altitude. Em Boa vista, o experimento foi instalado no mês de maio e, em Lavras, na primeira quinzena do mês de novembro do ano de 2000. O delineamento experimental utilizado foi o de látice simples $12 \times 12$, envolvendo os 135 híbridos de linhagens $S_{2}$, as gerações $\mathrm{F}_{1}$ dos híbridos simples, AG-9012, C-333B e Z-8392, o híbrido duplo C-435 e cinco outros híbridos intervarietais. A parcela foi composta por uma linha de $3 \mathrm{~m}$, espaçada de $90 \mathrm{~cm}$ entre linhas, semeando-se dez sementes por metro linear, objetivando deixar cinco plantas por metro após o desbaste. O tamanho da parcela foi definido em função do número de sementes disponível dos cruzamentos, observando a implantação do experimento em dois locais. A adubação de plantio foi de $450 \mathrm{Kg}$ da fórmula comercial 08-28-16 (NPK) + Zn. As adubações de cobertura foram realizadas aos 25 e aos 45 dias após o plantio, utilizando, como fonte de nitrogênio, sulfato de amônio (20\%), nas dosagens de 40 $\mathrm{Kg}$ de $\mathrm{N}$ por aplicação. O controle de plantas daninhas foi feito com herbicida pós-plantio auxiliado por capinas manuais. $\mathrm{O}$ controle de pragas foi feito aos 25 dias após o plantio, com inseticida piretróide, com o objetivo de controlar a lagarta-do-cartucho Spodoptera frugiperda (J. E. Smith, 1797) (Lepidoptera: Noctuidae). Os caracteres avaliados foram: produção de espigas despalhadas (g/parcela) (PESP), altura de plantas (cm) (ALTP) e altura de espigas(cm) (ALTE). Os dados referentes ao peso de espiga despalhada foram corrigidos para umidade padrão de $13 \%$, antes de serem submetidos à análise de variância.

\section{RESULTADOS E DISCUSSÃO}

Realizaram-se inicialmente análises de variância individuais para cada caráter baseada nos locais. Nessas análises, a precisão experimental, baseada nos coeficientes de variação, oscilou entre 14,36 a 18,14\% para PESP, de 5,97 a $6,86 \%$ para ALTP e de 9,64 a 14,24\% para ALTE. Esses valores podem ser considerados adequados para a cultura do milho (GOMES, 1990; SCAPIM et al., 1995).

Com o objetivo de conhecer a interação dos híbridos com os ambientes, de posse das análises individuais, foi realizada a análise conjunta envolvendo os dois locais, segundo Ramalho et al. (2000).

Constataram-se diferenças entre os híbridos de linhagens $\mathrm{S}_{2}$ para produção de espigas despalhadas. Verificou-se também que a fonte de variação testemunhas foi significativa. Isso evidencia que houve comportamento diferente das testemunhas. Um fato marcante é a nãosignificância do contraste híbridos vs testemunhas (Tabela 1), o que mostra que o comportamento médio dos híbridos 
de famílias $S_{2}$ foi semelhante ao das testemunhas. Na Figura 1 , encontram-se a distribuição de frequiências das médias para PESP e um quadro interno mostrando as produtividades médias das quatro testemunhas. Nota-se que a amplitude de variação foi bastante grande, de 4,22 a 12,88 tha na média dos dois locais. Considerando ainda o PESP, $81 \%$ dos híbridos $\mathrm{S}_{2}$ foram superiores às médias das testemunhas. Observou-se que $17 \%$ dos híbridos $S_{2}$ tiveram desempenho superior ao híbrido simples C333B (10,76 t/ha), que foi a testemunha com melhor desempenho e $46 \%$ foram superiores à testemunha C435 (9,56 t/ha), um híbrido duplo, amplamente utilizado nos dois Estados. Resultados semelhantes foram mencionados por Dantas (1992), na avaliação de 300 híbridos interpopulacionais em dez experimentos; em 6 dos 10 locais obtiveram-se médias superiores dos híbridos interpopulacionais em relação à testemunha (Cargill - 511).

$\mathrm{Na}$ decomposição das fontes de variação, capacidade geral de combinação (CGC), capacidade específica de combinação (CEC), verificou-se que tanto a CGC como a CEC foram significativas (Tabela 1). Esse fato mostra ser possível selecionar famílias $\mathrm{S}_{2}$ com grande CGC e selecionar famílias que se complementam por meio da CEC.
A distribuição da CEC (Figura 2 ) que variou de 455,47 a 377,85 para o caráter PESP indicou uma variação de $-17,76 \%$ a $14,74 \%$ em relação à média dos respectivos híbridos de linhagens $S_{2}$. Resultados semelhantes foram encontrados por Dantas (1992), que trabalhando com híbridos intermediários, encontrou variação entre $-13,9 \%$ a $11,85 \%$ da média geral para o mesmo caráter.

Foram verificadas também a ocorrência de interações das fontes híbridos, CGC e CEC com os ambientes. Essa forte interação também foi descrita por Araújo (1995), em que a CGC oriunda de um mesmo número de linhagens, em termos médios, um conjunto de híbridos simples, apresenta maior interação com o ambiente do que um conjunto de híbridos duplos (homeostase), ocorrendo o mesmo para a CEC devido a essa ser mais relacionada com cruzamentos específicos e efeitos de dominância. Esse fato dificulta a seleção de um híbrido para vários locais, porque os materiais avaliados não apresentaram um comportamento consistente nos locais estudados, ou seja, há uma mudança na classificação dos materiais nos locais avaliados. Assim, o ideal seria conduzir um programa de melhoramento específico para cada região, explorando essa interação em favor do melhoramento. Nesse caso particular, como os ambientes são muito contrastantes, deve-se prevalecer um programa para cada região.

TABELA 1 - Resumo da análise de variância conjunta de Lavras (MG) e Boa Vista (RR) para peso de espigas despalhadas (PESP) em gramas por parcela, altura de plantas ( ALTP) e altura de espigas (ALTE) em centímetros.

\begin{tabular}{|c|c|c|c|c|c|c|c|}
\hline \multirow[b]{3}{*}{ Cultivares (C) } & \multicolumn{7}{|c|}{ Quadrados Médios } \\
\hline & \multirow{2}{*}{$\frac{\text { GL }}{143}$} & \multicolumn{2}{|l|}{ PESP } & \multicolumn{2}{|c|}{ ALTP } & \multicolumn{2}{|c|}{ ALTE } \\
\hline & & 584163.42 & $* *$ & 717.75 & $* *$ & 799.08 & $* *$ \\
\hline Híbridos $(\mathrm{H})$ & 134 & 598113.43 & $* *$ & 734.91 & $* *$ & 820.73 & $* *$ \\
\hline CGC & 89 & 655363.06 & $* *$ & 882.57 & $* *$ & 1081.79 & $* *$ \\
\hline CEC & 45 & 484865.60 & $* *$ & 443.09 & $* *$ & 304.79 & ns \\
\hline Locais (L) & 1 & 28874502.25 & $* *$ & 66435.06 & $* *$ & 17622.56 & $* *$ \\
\hline $\mathrm{C} \times \mathrm{L}$ & 143 & 380683.01 & $* *$ & 288.36 & $*$ & 19.29 & ns \\
\hline Testemunhas $(\mathrm{T})$ & 8 & 399350.00 & $*$ & 518.94 & $*$ & 534.82 & $*$ \\
\hline $\mathrm{H}$ vs $\mathrm{T}$ & 1 & 193369.50 & $\mathrm{~ns}$ & 8.27 & ns & 11.93 & ns \\
\hline $\mathrm{H} \times \mathrm{L}$ & 134 & 1415164.78 & $* *$ & 1195.17 & $* *$ & 1238.02 & $* *$ \\
\hline CGC x L & 89 & 804663.91 & $* *$ & 587.94 & $* *$ & 633.51 & $* *$ \\
\hline CEC $x$ L & 45 & 515576.71 & $* *$ & 616.66 & $* *$ & 590.32 & $* *$ \\
\hline Erro & 254 & 184481.14 & & 226.44 & & 227.39 & \\
\hline $\mathrm{CV}(\%)$ & & 16.31 & & 6.47 & & 12.01 & \\
\hline Média & & 2564.18 & & 230.78 & & 124.94 & \\
\hline
\end{tabular}

Ciênc. agrotec., Lavras. V.27, n.5, p.985-990, set./out., 2003 


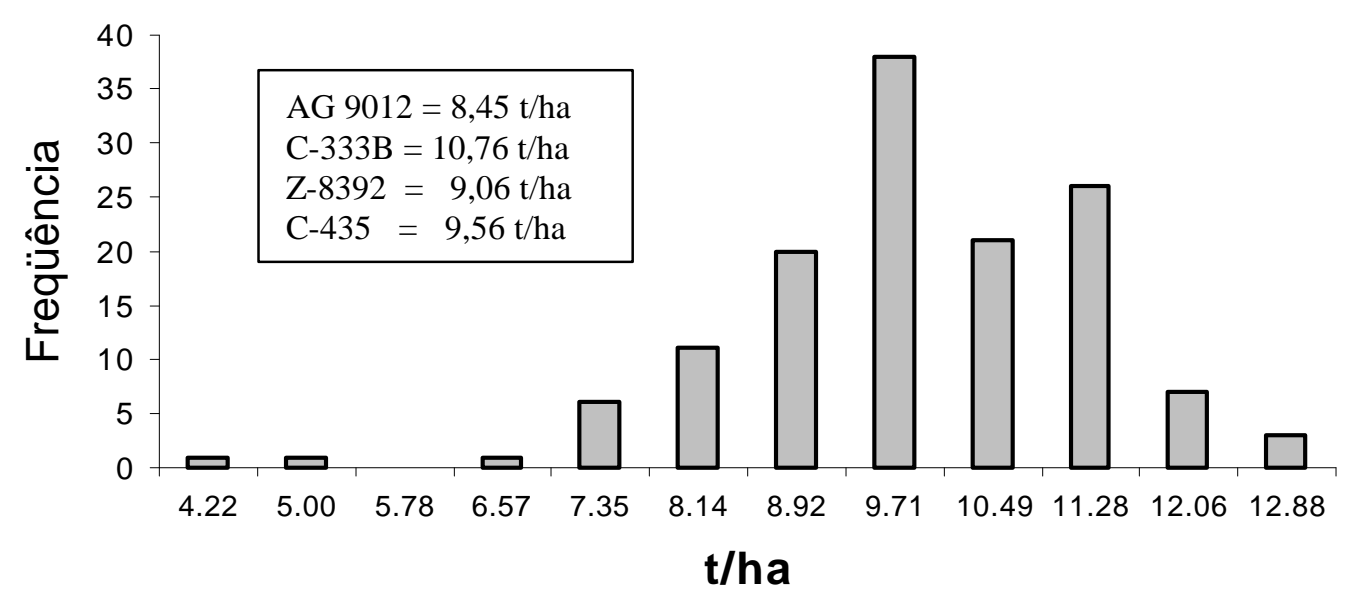

FIGURA 1 - Distribuição de freqüências na avaliação de híbridos $S_{2}$ nos Estados de Roraima e Minas Gerais para produtividade média de espigas despalhadas (t/ha).

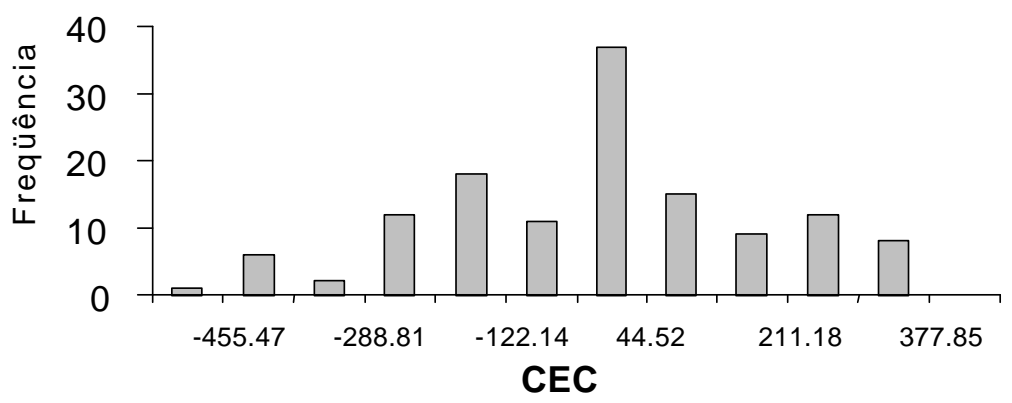

FIGURA 2 - Distribuição de frequiências obtidas na avaliação de híbridos $S_{2}$ nos Estados de Roraima e Minas Gerais para CEC, considerando o peso de espigas despalhadas ( $\mathrm{t} / \mathrm{ha})$.

Para ALTP e ALTE, os resultados foram semelhantes a PESP, salientando que o contraste $H$ vs $T$ foi não-significativo, indicando que os híbridos $\mathrm{S}_{2}$ e as testemunhas apresentaram em média as mesmas ALTP e ALTE. Embora os resultados médios não se mostraram significativos, houve um comportamento médio superior dos híbridos $\mathrm{S}_{2}$ em relação às testemunhas. No entanto, $17,06 \%$ e $38,71 \%$ dos materiais avaliados tiveram respectivamente ALTP e ALTE inferiores aos das testemunhas. Esses resultados são semelhantes aos encontrados por Dantas (1992), que citou a possibilidade de seleção, visando a reduzir ALTP e ALPE dentro desses híbridos, caso seja esse o objetivo do melhoramento.

\section{CONCLUSÕES}

a) Os híbridos de linhagens $S_{2}$ apresentaram alto potencial produtivo e altura de plantas e de espigas semelhantes às testemunhas. b) Híbridos de linhagens $S_{2}$ podem substituir com vantagens alguns híbridos comerciais disponíveis no mercado.

c) A grande variabilidade genética para peso de espiga despalhada dos híbridos de linhagens $\mathrm{S}_{2}$ possibilitou a seleção das progênies para desempenhos superiores em cruzamentos futuros.

d) O grande número de combinações híbridas superiores às testemunhas evidenciaram o grande potencial desse tipo de híbrido como concorrente direto de híbridos comerciais.

\section{REFERÊNCIAS BIBLIOGRÁFICAS}

ARAÚJO, J. S. de. Ganhos genéticos obtidos em híbridos e variedades de milho representativos de três décadas de melhoramento no Brasil. 1995. 64 f. Dissertação (Mestrado em Genética e Melhoramento de Plantas) - Universidade Federal de Lavras, Lavras, 1995. 
CARLONE, M. R.; RUSSEL, W. A. Evaluation of $\mathrm{S}_{2}$ maize lines reproduced from several generation by random mating within lines: 1 . comparations between the original and maintained $\mathrm{S}_{2}$ lines. Crop Science, Madison, v. 28, n. 6, p. 916-20, Nov./Dec. 1988.

CRUZ, C. D.; REGAZZI, A. J. Modelos biométricos aplicados ao melhoramento genético. 2. ed. Viçosa: UFV, 1997. 390 p.

DANTAS, J. L. L. Cruzamentos dialélicos parciais para avaliação de híbridos intermediários entre duas populações de milho (Zea mays L.). 1992. 103 f. Tese (Doutorado em Genética e Melhoramento de Plantas) - Escola Superior de agricultura Luiz de Queiroz, Piracicaba, 1992.

DAVIS, R. L. Report of plant breeder. Mayaguez: Puerto Rico Agricultural Experimet Station, 1927.

DUVICK, D. N. Maize breeding: past, present and future. In: CONGRESSO NACIONAL DE MILHO E SORGO, 20., 1994, Goiânia. Anais... Goiânia: CNPMS/EMBRAPA, 1994. p. 42.

GOMES, F. P. Curso de estatística experimental. 13. ed. Piracicaba: Nobel, 1990. 430 p.

JENKINS, M. T.; BRUNSON, A. M. Methods of testing inbreed lines of maize in crossbred combinations. Journal of American Society of Agronomy, Madison, v. 24, n. 7, p. 523-530, 1932.

LOEFFEL, F. A. $\mathrm{S}_{1}$ crosses compared with crosses of homozygous lines. In: CORN AND SORGHUM INDUSTRY RESEARCH CONFERENCE, 19., 1964, Chicago. Proceedings... Washington: AMERICAN SEED TRADE ASSOCIATION, 1964. p. 95-104.

MEDINA, S. A. V. Avaliação de híbridos simples de milho (Zea mays $L$.) obtidos de linhagens com diferentes graus de endogamia. 1990. 210 f. Dis- sertação (Mestrado) - Escola superior de Agricultura "Luiz de Queiroz", Universidade de São Paulo, Piracicaba, 1990.

MIRANDA FILHO, J. B.; VIÉGAS, G. P. Milho híbrido. In: PARTENIANI, E.; VIÈGAS, G. P. (Eds.). Melhoramento e produção de milho. Campinas: Fundação Cargill, 1987. v. 1, p. 275-340.

MOTA, M. G. C. Potencialidade de um novo sistema de cruzamento a nível interpopulacional, com progênies de meios irmãos de milho (Zea mays L.). 156 f. Tese (Doutorado em Genética e Melhoramento de Plantas) - Escola Superior de Agricultura "Luiz de Queiroz”, Piracicaba, 1984.

RUSSEL, W. A. Genetic improvement of maize yields. Advances in Agronomy, Ames, v. 46, p. 245-299, 1991.

RAMALHO, M. A. P.; FERREIRA, D. F.; OLIVEIRA, A. C. Experimentação em genética e melhoramento de plantas. Lavras: UFLA, 2000. 326 p.

SCAPIM, C. A.; CARVALHO, C. G. P.; CRUZ, C. D. Uma proposta de classificação dos coeficientes de variação para a cultura do milho. Pesquisa Agropecuária Brasileira, Brasília, v. 30, n. 5, p. 683-686, maio 1995.

SOUZA JÚNIOR, C. L. Variâncias genéticas interpopulacionais e suas relações com a obtenção e seleção de híbridos. 1998. 140 f. Tese (Livre docência) - Escola Superior de Agricultura “Luiz de Queiroz”, Piracicaba, 1998.

STANGLAND, G. R.; RUSSEL, W. Variability within single cross of $S_{2}$ and $S_{8}$ inbred lines of maize. Maydica, Bergamo, v. 26, n. 4, p. 227-238, 1981.

WELHAUSEN, E. J. Modern corn breeding and production in Mexico. Phytopatology, Saint Paul, v. 44, n. 8, p. 391-395, Aug. 1954. 